\title{
GAS DYNAMICS IN THE CENTRAL PART OF THE SY 1 GALAXIES: III ZW 2 AND MRK 817
}

\author{
L. Č. POPOVIĆ, I. VINCE, A. KUBIČELA AND S. SALIM \\ Astronomical Observatory, Volgina 7, 11000 Belgrade, Serbia, \\ Yugoslavia
}

\section{Introduction}

Spectra of Active Galactic Nuclei (AGN) indicate that in their central parts a composite motion of emission gas exists. An analysis of broad as well as narrow lines of active galaxies can give information about gas dynamics in central part of these objects (see e.g. Netzer 1990, Osterbrock 1990). Here we present the investigation of the $H_{\beta}$ and $O$ III[4959,5007] line shapes of the two Sy 1 galaxis: Mrk 817 and III Zw2.

The spectra were obtained at $2.6 \mathrm{~m}$ telescope of Crimean Astrophysical Observatrory by K.K. Chuvaev during the period 1971-1991 (for details see Popović 1996). The spectral regions covered $H_{\beta}$ and $O$ III $(4959,5007)$ emission lines. There is no significant short time variability of the Mrk 817 and III $\mathrm{Zw} 2$ spectra, so, we averaged the spectra for one or two year periods, namely 3 and 8 for the former and later galaxy respectively. Here we present only the mean velocities $(\langle V\rangle)$ for all the observed spectra.

\section{Method of analysis and results}

We suppose that the $H_{\beta}$ in these galaxies is composed by two or more components, e.g. that emission from two or more regions can be seen in $H_{\beta}$. So, we have fitted this wavelength region $\left(H_{\beta}\right.$ and $O$ III $\left.[4959,5007]\right)$ with several gaussians.

In order to avoid too many input parameters and to reduce the errors in the obtained gaussian fit parameters, we have included some restrictions: 1 . Taking into account that both of the narrow $\mathrm{O}$ III lines orginated from the same multiplet (1F) and that the lines are formed in the same (NLR) region their widths are in the next relationship $\Delta W_{4959} / 4959=\Delta W_{5007} / 5007$, 
where $\Delta W_{\lambda}$ is line width of the line. One of the components of the $H_{\beta}$ is formed in NLR and line width of this component follows the same relation and has the same shift as $\mathrm{O}$ III lines. 2. Also, we take that for $\mathrm{O}$ III lines $I_{\lambda} \sim S_{\lambda}$. Then the ratio of their intensity have to be $I_{5007} / I_{4959} \approx 3.03$. The dispersion velocities of gas in regions are calculated as $V[\mathrm{~km} / \mathrm{s}]=$ $(2 \cdot \Delta W) /\left(2.31 \cdot \lambda_{0}\right) \cdot c$, where $\Delta W$ is the half-width of gaussian function for the corresponding region, $\lambda_{0}$ is the rest wavelength and $c$ is the speed of light.

In our explanation of $H_{\beta}$ and $O$ III line shapes of Mrk 817 we prefer the model with gas outflows in the BLR, ILR and partly in NLR. Then we can explain the red asymmetry of broad $H_{\beta}$ line as caused by gravitational redshift (Mediavilla \& Insertis 1989, Atanacković-Vukmanović et al. 1994, Corbin 1995, 1997 and Popović et al. 1994,1995). The broadest line of the emitting gas comes from the region of strong gravitation field that can cause a part of the observed redshift. It produces a strong red asymmetry of the $H_{\beta}$ line profile. Also, an outflow of the emitting gas is present. In the case of this galaxy, dispersion velocities, $\langle V\rangle$, of the emitting gas in the regions are: in BLR $7600 \mathrm{~km} / \mathrm{s}$, in ILR $2200 \mathrm{~km} / \mathrm{s}$ and in NLR $460 \mathrm{~km} / \mathrm{s}$.

In the case of the III $\mathrm{Zw} 2$ we found that two regions exist: BLR with $\langle V\rangle=3100 \mathrm{~km} / \mathrm{s}$ and $N L R$ with $\langle V\rangle=500 \mathrm{~km} / \mathrm{s}$. The Broad Line Component of $H_{\beta}$ is double peaked indicating that an emitting disk is present here. It is in agreement with the observation of continuum distribution (see Kaastra and de Korte 1988), where was noted that an increase of the energy density in the optical part of spectrum $\left(\approx 10^{15}\right)$ can be explained as radiation of a backbody disk. Hence, we a priori suppose that the two broad gaussians have the same width and intensity.

\section{References}

Atanacković-Vukmanović O., Popović L. Ċ., Vince I. \& Kubičela A., 1994, Bull. Astron. Belgrade 150, 1.

Corbin M. R., 1995, Astrophys. J. 447, 496.

Corbin M. R., 1997, Astrophys. J., in press

Kaastra J. S., de Korte P. A. J. Astron. Astrophys. 198, 16.

Mediavilla E. \& Insertis F. M., 1989, Astron. Astrophys. 214, 79.

Netzer H., 1990, in Active Galactic Nuclei, eds. R. D. Blandford, H. Netzer \& L. Woltjer, Saas-Fee Advanced Course 20, Berlin: Springer - Verlag.

Osterbrock D. E., 1989, Astrophysics of Gaseous Nebulae and Active Galactic Nuclei, Mill Valley, California.

Popović L. Č., 1996, Publ. Astron. Obs. Belgrade 54, 49.

Popović L. Č., Vince I., Kubičela A., Atanacković-Vukmanović O. \& Samurović S., 1994, Bull. Astron. Belgrade 149, 9.

Popović L. Č., Vince I., Atanacković - Vukmanović O. \& Kubičela A., 1995, Aston. Astrophys. 293, 309. 